\title{
Changes in inter-intromission interval during uninterrupted copulation in rats'
}

DONALD A. DEWSBURY

UNIVERSITY OF FLORIDA

Data from 79 male rats in 416 copulation tests with 4413 Inter-Intromission Intervals (III) were analyzed in order to determine whether III changes within the copulatory series that leads to ejaculation. III increases from its initial value and then decreases regularly as ejaculation is approached.

In the typical copulatory pattern of rats a series of mounts with and without vaginal intromission precedes the ejaculatory intromission. Little is known of changes in the behavior or physiological state of rats during the period of copulation as ejaculation is approached. One behavioral measure that might be expected to change between the beginning of copulation and ejaculation is the Inter-Intromission Interval. Inter-Intromission Interval (III) may be defined as the interval between two successive intromissions within a preejaculatory series of mounts and intromissions. Clemens (1966) has shown that in the first ejaculatory series of the deermouse, Peromyscus maniculatus Gambeli, the mean III shortens in successive quarters of the ejaculation latency period (that period between the first intromission and ejaculation). The purpose of the present study is to search for changes in III in laboratory rats during uninterrrupted copulation. Such changes would give some indication of the state of the rat during the pre-ejaculatory period.

Due to the necessity of amassing an appreciable amount of data so that curves of reasonable stability might be obtained, the data from five separate experiments were used. One of the experiments was run expressly to provide data for the present analysis, but the remainder were run for various other purposes. Because the effect under study involves changes within tests, many procedural variations would be expected to have little effect on the results. All experiments had the following characteristics in common: (1) Long-Evans rats were used, (2) all rats had copulatory experience prior to testing, (3) the results of at least two tests per animal were available, and (4) all tests were continued until the first intromission after ejaculation. Data from a total of 79 male rats in 416 copulation tests with 4,413 IIIs were subjected to analysis.

Method

A total of 79 males and approximately 130 females were used in the studies which provided data for the present analysis. All males were at least 90 days of age at the beginning of study with a range of 90-180 days. All males had copulatory experience prior to the beginning of testing with a range of 1 to 20 prior ejaculations. Sixty of the Long-Evans rats were obtained from a commercial supplier, while the remainder were obtained from the colony maintained by the Department of Anatomy of the University of California, Berkeley.

Forty-five of the animals were tested in three identical arenas with walls of Plexiglas and floors of wood covered with sawdust. They were circular with diameters of 36 in. and heights of 30 in. Thirty-four animals were tested in semi-circular, internally-lighted observation cages made of metal painted a flat gray and with Plexiglas fronts. They had internal radii of 18 in. and wee 15-1/2 in. high. Behavioral events were recorded on an Esterline-Angus event recorder.

The males were given 2-16 tests spaced 3-14 days apart. On each test the male was placed in the mating arena 3-5 $\mathrm{min}$. before the female. The test began with the introduction of the female and continued to the first intromission following ejaculation. Thus all data refer to the first ejaculatory series.

All females were brought into estrus with exogenous hormone treatment. For all but six the standard procedure of giving a $0.1 \mathrm{mg}$ intramuscular injection of estradiol benzoate $72 \mathrm{hr}$. before testing and $1.0 \mathrm{mg}$ of progesterone $3 \mathrm{hr}$. before testing was used. ${ }^{2}$ The

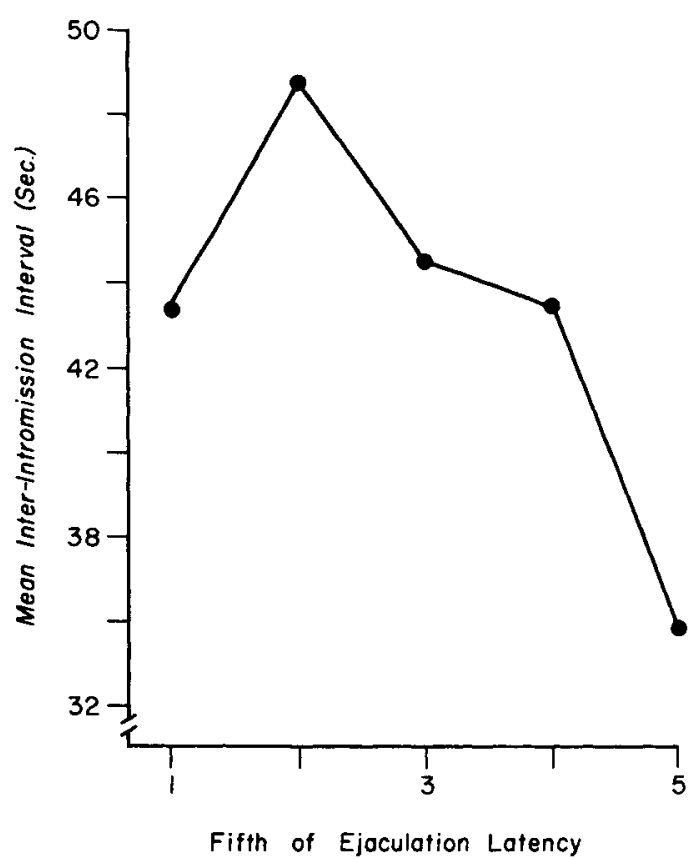

Fig. 1. Mean Inter-Intromission Interval in Successive Fifths of the Ejaculation Latency Period ( $N=79$ ). 
Table 1. Number of Rats Showing Mean Increase, Decrease, and No Change in III in Relevant Comparisons.

\begin{tabular}{|c|c|c|c|c|c|}
\hline Comparison & \multicolumn{3}{|c|}{ Direction of Shift } & $\mathbf{z}$ & $p$ \\
\hline \multicolumn{6}{|c|}{ Fifths of EL: } \\
\hline Ist vs. 2nd & 56 & 23 & 0 & 3.64 & $<.0003$ \\
\hline 2nd vs. 3rd & 30 & 49 & 0 & 2.05 & $<.04$ \\
\hline 3 rd vs. 4 th & 41 & 37 & 1 & 0.34 & N.S. \\
\hline 4 th vs. 5 th & 16 & 62 & 1 & 5.22 & $<.00006$ \\
\hline \multicolumn{6}{|c|}{ Individual $1 / 1$ s: } \\
\hline 1 vs. 2 & 63 & 15 & 1 & 5.24 & $<.00006$ \\
\hline 2 vs. 3 & 43 & 36 & 0 & 0.68 & N.S. \\
\hline N-2 vs. N-1 & 42 & 37 & 0 & 0.46 & N.S. \\
\hline N-l vs. N & 21 & 55 & 3 & 3.79 & $<.0002$ \\
\hline
\end{tabular}

remaining six females were ovariectomized and implanted with crystalline pellets of estrogen

Resulfs

In all analyses data from each rat were equally weighted regardless of the number of tests run. Each ejaculation latency period (EL) was divided into fifths and each III classified according to the fifth of the EL in which it began. Means for subjects and for the entire study were then calculated. The mean IIIs for the entire study (with Ss equally weighted) in successive fifths of the EL are given in Fig. 1. Mean III appears to increase from the first to the second fifth and to decrease after that. The data were analyzed according to the number of rats which showed increases, decreases, and no change in mean III for all tests run. Friedman two-way analysis of variance (Siegel, 1956) revealed a significant difference among the five fifths of the $E L\left(X^{2}=62.2, p<.001\right)$. Results of subsequent sign tests (Siegel, 1956) on individual comparisons of fifths of the EL are given in Table 1. The changes in III which appear appreciable in the figure are statistically significant. When data from separate sources were analyzed separately, no contradictions to these trends were found. Thus, when data are classified according to the fifth of the EL in which each III began, III appears to increase from its initial level and to decrease regularly after that.

Because of the possibility that this effect might be due to the method of grouping IIs, data from individual IIIs were analyzed separately. Due to the relatively small number of intromissions in some tests only data from the first and last three IIIs could be so analyzed. The results are presented in the bottom of Table 1. The shifts from the first to the second III (Means 38.9 to $47.1 \mathrm{sec}$.) and from the $n-1$ th to the pre-ejaculatory III (Means 44.3 to 36.2 sec.) were highly significant, the former showing an increase and the latter a decrease. It would appear that much of the effect seen in Fig. 1 is due to the shifts from the first and to the last short III. The fundamental effect is not an artifact of the grouping of data as in Fig. 1. When data from the one study run expressly for this analysis are examined, all major effects are present and statistically significant.

\section{Discussion}

The data from this study provide one of the few indications of a change in the behavior of rats within a series leading to ejaculation. The rats start the series copulating at a moderate rate, slow down, and then speed up as ejaculation is approached. Interpretation of this effect is difficult. A simple acceleration as ejaculation is approached might be regarded in the context of a buildup of excitation in an hypothetical copulatory mechanism (Beach, 1956) or as a goal gradient during the approach (in time) toward ejaculation (Hull, 1932). With the initial deceleration, however, neither of these interpretations is convincing. There is an acceleration of copulatory rate as ejaculation is approached in both deermouse (Clemens, 1966) and rat, but the initial deceleration following the first III appears only in the rat. This effect must be considered in models of the control of copulation in male rats though a convincing theoretical interpretation is not possible at this time.

\section{References}

Beach, F. A. Characteristics of masculine "sex drive". In M. R. Jones (Ed.), Nebraska symposium on motivation. Lincoln: University of Nebraska, 1956.

Clemens, L. G. Mating behavior of the deermouse, Peromyscus maniculatus Gambeli. Doctoral dissertation, University of California, Berkeley, 1966.

Hull, C. L. The goal gradient hypothesis and maze learning. Psychol. Rev., 1932, 39, 25-43.

Siegel, S. Nonparametric statistics for the behavioral sciences. New York: McGraw-Hill, 1956.

\section{Notes}

1. This research was done during the author's tenure as a postdoctoral fellow of the National Science Foundation in the Department of Psychology of the University of California, Berkeley, The work facilitated by grant MH 04000-07 from the National Institutes of Health to Dr. Frank A. Beach. The author wishes to thank Mr. William Westbrook of the University of California, Berkeley for providing the data from nineteen rats

2. The hormones used were Progynon brand of estradiol benzoat and Proluton brand of progesterone supplied through the courtesy of Dr. Preston L. Perlman of the Schering Corporation, Bloomfield, New Jersey. 